\title{
Common and distinct neural responses during direct and incidental processing of multiple facial emotions
}

\author{
J.S. Winston,* J. O’Doherty, and R.J. Dolan \\ Wellcome Department of Imaging Neuroscience, 12 Queen Square, London WCIN 3BG, UK
}

Received 4 February 2003; revised 10 March 2003; accepted 1 May 2003

\begin{abstract}
Whether common or distinct neural systems underpin perception of different emotions and the degree to which these systems are automatically engaged during emotional perception are unresolved. We performed an event-related fMRI experiment in which subjects viewed morphed emotional faces displaying low or high intensities of disgust, fear, happiness, or sadness under two task conditions. The amygdala and fusiform cortex responded to high-intensity expressions of all emotions, independent of task. Right superior temporal sulcus showed an additive effect of the emotion-directed task and high-intensity emotion. Ventromedial prefrontal and somatosensory cortices, regions implicated in providing representations of somatic states, showed enhanced activity during explicit emotional judgments. We failed to find predicted differences between emotions. The results suggest that amygdala contributes to task-independent perceptual processing of a range of emotions. We interpret ventromedial prefrontal and somatosensory cortex activations as evidence that these regions contribute to explicit emotion processing through linking emotion perception with representations of somatic states previously engendered by emotions.
\end{abstract}

(C) 2003 Elsevier Inc. All rights reserved.

\section{Introduction}

Social cognition reflects an ability to recognize and respond appropriately to emotion in others (Adolphs, 1999; Brothers, 1990). A pancultural means of signaling emotion is through facial expression (Darwin, 1872; Ekman, 1993) and the successful interpretation of facial expressions of emotion has been considered to constitute an innate, nonverbal, automatic process (Darwin, 1872; Ohman, 2002). An influential contemporary theory of human emotion, the "somatic marker hypothesis," proposes an intimate association between bodily and brain states in the perception and experience of emotion (Damasio, 1994, 1999).

The somatic marker hypothesis has been extended in a recent proposal that perception of an emotion in a conspecific involves simulation of the emotional state within the relevant cortical circuitry of the observer (Adolphs et al., 2000; Adolphs, 2002a, 2002b). Support for this draws largely upon evidence from studies of patients with discrete

* Corresponding author. Fax: +44-0-20-7813-1420.

E-mail address: j.winston@ fil.ion.ucl.ac.uk (J.S. Winston). brain lesions. In one of these studies, patients with ventral prefrontal lesions showed deficits in recognition of emotion from faces and voices relative to patients with lesions at other sites (Hornak et al., 1996). Ventral prefrontal cortex has long been implicated in the generation and mapping of somatic markers (summarised in Damasio, 1994, 1999). A study of a larger sample of patients suggested that right somatosensory cortices were particularly critical to this function (Adolphs et al., 2000). One problem in relation to these findings is that neuroimaging studies have generally failed to demonstrate activation in these regions during facial emotion perception. A possible reason is that human neuropsychological studies might conflate inability to perform the recognition task with impaired perception of facial emotion.

A further issue in the study of emotion perception is whether different brain regions process distinct emotions. Recent neuropsychological and neuroimaging data have been interpreted as indicating that emotional perception, and perception of facial expressions in particular, is organized in a modular fashion with distinct neural circuitry subserving individual emotions (Adolphs, 2002a; Calder et 
al., 2001). However, examination of these data suggests that this conclusion may be premature, and whether there is a common substrate to the perception of multiple basic emotions has not been systematically investigated using noninvasive neuroimaging techniques.

Much early research into brain mechanisms for emotional perception involved either single unit recording in non-human primates or neuropsychological studies of patients with focal lesions. Neurophysiological evidence suggests that circumscribed brain regions contain neurons responsive to facial movements and specific facial expressions, and that these neurons are segregated from those responding to facial identity (Hasselmo et al., 1989; Rolls, 1992). Lesion studies in humans report two distinct findings: certain lesions impair recognition of all so-called "basic emotions" (Adolphs et al., 1996, 2000; Hornak et al., 1996), whereas lesions in other brain areas impair recognition of a more limited subset of facial emotions (Adolphs et al., 1994, 1995; Calder et al., 1996; Sprengelmeyer et al., 1999). Thus, ventral prefrontal and right cortical lesions result in a generic impairment in facial emotion recognition as measured in group studies (Adolphs et al., 1996, 2000; Hornak et al., 1996; Kolb et al., 1983; Rapcsak et al., 1993). As indicated above, right somatosensory cortex has been highlighted as a critical locus in facial emotion recognition that spans different emotions in the largest group study to date (Adolphs et al., 2000). Single-subject data, by contrast, have focused on consequences of damage to distinct brain regions causing impairment in recognition of specific emotions. For example, impairment in fear recognition is reported following bilateral damage to the amygdala (e.g., patients NM, RB, SE, SM, and YW-Adolphs et al., 1994, 1995; Broks et al., 1998; Calder et al., 1996; Sprengelmeyer et al., 1999), but the selectivity of this deficit remains unclear insofar as patients are commonly impaired on recognition of more than one emotion (e.g., patients DR, GP, JC, JM, NM, and SP-Adolphs et al., 1999; Broks et al., 1998; Calder et al., 1996; Rapcsak et al., 2000; Schmolck and Squire, 2001; Young et al., 1995, 1996), and occasionally do not manifest fear recognition deficits (e.g., patients DBB, EP, GT, and RH-Adolphs et al., 1999; Hamann et al., 1996). Additionally, a single patient with discrete lesions in left insula and basal ganglia has been described as showing a specific deficit in disgust recognition (and experience) (Calder et al., 2000). Although a double dissociation between neural substrates of recognition for fear and disgust is of considerable theoretical interest (Calder et al., 2001), caution has been urged on the basis that individual differences between subjects and task difficulty may confound inferences from single case studies (Adolphs, 2002b; Rapcsak et al., 2000).

A powerful means of studying the neural correlates of emotion perception in the intact human brain is afforded by functional neuroimaging. To date, studies indicate that discrete brain regions process human facial emotions even when facial emotion perception is not task relevant, con- comitant with the idea that facial expression is processed automatically (Blair et al., 1999; Breiter et al., 1996; Dolan et al., 1996; Morris et al., 1996; Phillips et al., 1997; Vuilleumier et al., 2001). Such findings raise the possibility that regions involved in perception of facial emotion may be dissociable from those involved in acting upon that information. This is an important consideration insofar as patient lesion studies reflect impairments on direct emotion recognition tasks. The most widely replicated neuroimaging finding is an amygdala response to fearful faces (Breiter et al., 1996; Dolan et al., 2001; Hariri et al., 2000; Morris et al., 1996; Phillips et al., 1997, 1998; Vuilleumier et al., 2001; Whalen et al., 1998). Facial expressions of disgust are reported to activate insula and basal ganglia, but not amygdala (Phillips et al., 1997). However, the claim that neuroimaging and lesion data have established dissociable neural substrates for fear and disgust (Calder et al., 2001) is open to question. For example, fearful faces are reported to activate insula and basal ganglia (Phillips et al., 1999), while disgust faces are reported to activate amygdala more than happy (Gorno-Tempini et al., 2001) and neutral faces (Phillips et al., 1998). In addition, comparing patterns of activation for disgust relative to neutral faces and for fearful relative to neutral faces with data derived from different studies does not provide a statistically convincing means of evaluating differences between emotions. Inferences regarding dissociable responses to distinct emotions require a direct comparison of different emotions within the same study, and few such studies have been performed. In one such study with six subjects, Phillips and colleagues reported that certain regions showed greater responses to disgust than to fear within left anterior insula and right putamen/globus pallidus (Phillips et al., 1998). In contrast, these authors also reported greater activation to fear than disgust in amygdala, although the coordinate for this activation corresponds better to anterior hippocampus than amygdala. Kesler-West and colleagues also examined responses to multiple emotions within one experimental paradigm and, using a region of interest approach, demonstrated dissociable responses to different emotional expressions (angry, fearful, happy, and sad) with a greater response to fearful faces in inferior frontal gyrus and to angry faces in medial frontal cortex (Kesler-west et al., 2001). Limitations of this study include the absence of a constrained experimental task and the use of a block design, with different emotions presented in different runs, rendering it impossible to separate effects of emotional expression from cognitive set and anticipatory effects engendered by block presentation.

The approach adopted in our experiment is to assess the effect of task (direct versus incidental processing) on neural responses to facial expressions when multiple emotions are considered within a single experiment. As highlighted above, the issue of task effects is critical in reconciling human lesion with functional imaging data. Lesion studies are limited to tasks requiring explicit recognition of facial 
emotion, whereas the majority of functional imaging studies have relied upon incidental emotional processing. A limitation of lesion studies is the possibility that they may conflate subjects' inability to utilize intact perception of facial emotion to successfully perform a task with an inability to accurately perceive the emotion. Recently, two imaging experiments tackled the question of task effects (Critchley et al., 2000a; Gorno-Tempini et al., 2001) although both were limited by blocked presentation of emotional stimuli. This entailed subjects making judgments of emotion ("emotional or nonemotional") within a block of either emotional or nonemotional faces, a suboptimal design. We adopted an optimal approach offered by event-related designs, wherein stimulus order is randomized such that subjects are unable to predict the next stimulus from the preceding one. In order to assess the neural correlates of making judgments concerning the emotional expression of faces, our design incorporated an "incidental" task in which subjects made a complex gender judgment concerning a pair of faces and a task in which subjects judged emotion in a pair of faces ("direct" task).

\section{Materials and methods}

\section{Subjects}

Twelve right-handed volunteer subjects (five female) consented to take part in the study, which was approved by the Joint National Hospital for Neurology and Neurosurgery/Institute of Neurology Ethics Committee. Subjects were free from medication, neurological or psychiatric history, and ranged in age from 22 to 41 . Data from one scanning session in one subject (a male) were lost due to technical failure, and as a consequence this subject's data were not included in the group analysis. The group analysis therefore consisted of data from 11 subjects (five female) with mean age of 26.

\section{Stimuli}

Stimuli were generated from facial photographs of eight individuals from the Ekman and Friesen series of emotional faces (Ekman and Friesen, 1976). To generate stimuli, we utilized commercially available morphing software (Morpher 2.0). Neutral faces of one gender were morphed toward emotionally expressive faces of the other gender. Stimuli were generated at 10,30, 70, and $90 \%$ between the two original images. The morphing procedure involved delineating anatomical loci and regions common to the two original images. In total, approximately 140 loci were delineated per face, to include the outline of the face, forehead wrinkles, the eyes and eyebrows, orbicularis occuli, the nose, zygomaticus muscles, and chin. Example stimuli are shown in Fig. 1A. Four emotions were used as target morphs: disgusted, fearful, happy, and sad faces. The iden- tities used to generate the faces were $\mathrm{C}, \mathrm{MO}, \mathrm{NE}, \mathrm{SW}$ (female) and EM, JJ, PE, WF (male).

Task

Subjects saw two faces either side of a fixation cross which remained on screen during the intertrial interval. Faces were either 10 and $30 \%$ or 70 and $90 \%$ morphs from the same series of images (Fig. 1A). We refer to trials containing 10/30\% morphs as "low-intensity" trials and trials consisting of 70/90\% morphs as "high-intensity" trials. Subjects' task during the experiment was blocked and alternated between choosing which face was more emotional or which face was more male (Fig. 1C). Faces appeared for $1.5 \mathrm{~s}$ and minimum stimulus onset asynchrony was $3 \mathrm{~s}$. Blocks consisted of 10 trials of one task followed by 10 trials of the other. Changes of block were indicated to subjects by means of a text display at the start of each block indicating which decision they were to make. Within each block, low- and high-intensity trial types of each emotion were randomly intermixed with one another and with null events. Scanning was divided into two 13-min sessions. Within each session 192 events (12 events of each type) were randomly interspersed with 48 null events. The total number of events of each type was therefore 24 . The first task block was counterbalanced across subjects.

Prior to scanning, subjects were shown examples of stimuli similar to those used during the scanning session and were trained on the experimental task.

\section{fMRI scanning}

Subjects were scanned during task performance using a Siemens VISION system (Erlangen, Germany) at $2 \mathrm{~T}$ to acquire gradient-echo, echoplanar $\mathrm{T} 2 *$-weighted images with blood oxygenation level dependent (BOLD) contrast. Each volume comprised $33 \times 3.3-\mathrm{mm}$ axial scans with 3 -mm in-plane resolution. For each of two sessions, 340 volumes were continuously acquired every $2.5 \mathrm{~s}$. Subjects were placed in light head restraint within the scanner to limit head movement during acquisition. To allow for T1 equilibration effects the first five volumes of each run were subsequently discarded. Additionally a T1-weighted structural image was acquired in each subject. These structural images were coregistered with the mean EPI from the functional acquisition (Collignon et al., 1995), normalized into a standard space using the normalization parameters applied to the EPIs and subsequently averaged for overlay of statistical parametric maps.

During scanning, eye position was monitored using an infrared eye tracker (ASL Model 450, Applied Science Group Co., Bedford, MA; refresh rate $=60 \mathrm{~Hz}$ ). These data were available for eight subjects. Due to technical failure, temporal resolution was inadequate for confident trial-bytrial analysis of eye position. However, we were able to compare eye position across the block component of the 
task. This was undertaken by defining a 25 -s time window and measuring the cumulative position along the horizontal axis in three locations approximating to the left face, right face, and fixation cross. Differences (between incidental and direct task) in cumulative time spent at each locus during were then entered into a series of one-sample $t$ tests.

\section{Data analysis}

Preprocessing of imaging data and statistical analysis was undertaken using SPM99 (Wellcome Department of Imaging Neuroscience; http://www.fil.ion.ucl.ac.uk/spm). All functional volumes were realigned to the first volume (Friston et al., 1995a), slice time corrected (Henson et al., 1999), normalized (Friston et al., 1995a) to an EPI template based upon the MNI reference brain in a standard space (Talairach and Tournoux, 1988) to allow group analysis and smoothed with an 8-mm FWHM Gaussian kernel to account for residual intersubject differences. The data were high pass filtered with a frequency of $1 / 120 \mathrm{~Hz}$.

For event-related analysis, delta functions corresponding to the onset of different event types were constructed and convolved with a synthetic hemodynamic response function (HRF) and its temporal derivative (Friston et al., 1998). Additionally, regressors corresponding to linear trends in response with time were constructed for each event type on the basis of the known-phenomenon of changes in the profile of responses to emotionally salient stimuli with time (e.g., Breiter et al., 1996; Buchel et al., 1998, 1999; Phillips et al., 2001). Subject-specific movement parameters were also used in the subsequent general linear model to take into account residual effects of subject motion. In a first level analysis parameter estimates for each regressor were calculated for each voxel (Friston et al., 1995b). Differential effects were tested for, treating the two sessions as one for all results reported here, and contrast images constructed, whereby the size of a given effect at each voxel constitutes the image. For random effects analysis (Friston et al., 1999), these images were entered into one-sample $t$ tests or ANOVAs as appropriate. Where ANOVAs were used, departures from sphericity assumptions were accommodated (Glaser et al., 2002): An iterative restricted maximum likelihood (ReML) procedure estimated hyperparameters controlling nonspherical variance components. These components were those induced by relaxing identity and independence assumptions about the errors. To ensure precise hyperparameter estimation, the error covariance was factorized into a voxel-specific hyperparameter and a voxel-wide correlation matrix. The correlations were estimated with ReML using all responsive voxels in the brain (i.e., those exceeding a criterion $F$ test for effects of interest at $P<0.001$ ) (Glaser et al., submitted). The ensuing error covariance at each voxel was used to compute (i) the appropriate statistic and (ii) the effective degrees of freedom according to the Satterthwaite approximation (formally equivalent to a Green-
house-Geisser correction), as described in Worsley and Friston (1995).

A global threshold was set at $P<0.001$ uncorrected for multiple comparisons. We report results that survive corrections for multiple comparisons across the whole brain at $P<0.05$, and $P<0.001$ uncorrected in regions predicted a priori. Additionally, we indicate whether activations in predicted regions survived a correction for multiple comparisons across small volumes of interest (Worsley et al., 1996). In right posterior STS, small volume correction (SVC) was based upon a sphere centered upon a previously established coordinate (12-mm radius; $x, y, z=56,-44,4)$ (Winston et al., 2002). In the cases of right SI and bilateral amygdala and insula masks based upon on the group's average structural scan were constructed using MRIcro (Rorden and Brett, 2000) (volumes 20,8, $40 \mathrm{~cm}^{3}$, respectively). For fusiform and face-responsive extrastriate cortex a mask derived from an independent study was used (faces versus fixation), extending from $-84 \mathrm{~mm}$ posteriorly to $-40 \mathrm{~mm}$ anteriorly, based on the maximum and minimum coordinates typically reported for face-specific activations (Haxby et al., 1999; Kanwisher et al., 1997; Vuilleumier et al., 2001) (volume $35 \mathrm{~cm}^{3}$ ).

\section{Results}

\section{Behavioral}

Participants' reaction times were analyzed by means of repeated-measures ANOVA using SPSS (SPSS, Inc., Chicago). This analysis revealed no main effect of task or intensity $(P>0.7)$, but a main effect of emotion was significant $(F(3,30)=2.95, P<0.05)$, clarified by a significant emotion-by-task interaction $(F(1.97,19.7)=10.05$ (Greenhouse-Geisser), $P<0.05$ ). This latter effect seemed driven by a facilitation effect in the direct task specific to happy faces, and a cost in the incidental task specific to fearful faces (Fig. 2).

Cumulative time spent looking at the left face, right face, and fixation cross were measured and compared across tasks for eight subjects for whom eye-tracking data were available. No significant difference between tasks was found for any of these three measures (left face: mean difference $=$ $-75.8 ; t(7)=-1.47 ; P=0.18$; right face: mean difference $=123.7 ; t(7)=1.40 ; P=0.20$; fixation cross: mean difference $=-152 ; t(7)=-1.78 ; P=0.11)$.

\section{Neuroimaging}

\section{Main effect of intensity}

The contrast of high versus low intensity expressions (collapsing across task and emotion type) showed activation in predicted regions: fusiform and extrastriate cortex $(x, y, z$ $=34,-72,-20, Z=3.99, P<0.1 \mathrm{SVC} ; x, y, z=$ $-36,-46,-24, Z=3.73, P<0.001$ uncorrected) and 
bilateral amygdata $(x, y, z=34,0,-26, Z=3.50, P<0.05$ SVC; $x, y, z=-24,-6,-18, Z=3.28, P<0.001$ uncorrected). To ensure that these activations were not a result of a disproportionate contribution from one task, we carried out a conjunction analysis (Price and Friston, 1997) of the contrast of high- minus low-intensity expressions across the two tasks, in effect testing the task independence of these responses. Significant bilateral amygdala and fusiform responses were evident in this contrast, confirming the taskindependence of these activations.

\section{Tests for similarities between emotions-conjunction analysis}

Although amygdala and face-responsive regions of extrastriate and inferior temporal cortex were activated by the main effect of intensity (see above), such analysis does not preclude the possibility of a predominant effect from one or more distinct emotions. We thus carried out a conjunction analysis across the contrasts of high-intensity minus lowintensity trials (collapsed across task) from each individual emotion type. In effect this conjunction analysis examines for a common effect across four contrasts, i.e., the effect of highly emotional faces, independent of subtype of emotion. This analysis revealed significant activations in bilateral amygdala $(x, y, z=30,-4,-22, Z=3.77, P<0.05 \mathrm{SVC}$; $x, y, z=-24,-2,-24, Z=3.24, P<0.001$ uncorrected) (Fig. 3) as well as fusiform and extrastriate regions $(x, y, z$ $=28,-66,-12, Z=4.57, P<0.05 \mathrm{SVC} ; x, y, z=$ $-30,-42,-20, Z=4.26, P<0.05 \mathrm{SVC}$ ) (Fig. 4). On the basis of this finding, the null hypothesis that at least one of these emotions failed to activate the amygdala can be rejected. A trend toward significance was evident in right posterior STS $(x, y, z=48,-46,4 ; Z=3.52 ; P<0.1 \mathrm{SVC})$ (Fig. 5).

It should be noted that this analysis collapses across tasks and does not therefore preclude the possibility that one task does not contribute to this effect of intensity. We thus carried out a further conjunction analysis across the eight contrasts representing high-intensity minus low intensity for each emotion category under each task condition. Even under these stringent conditions activation in left amygdala and bilateral fusiform and extrastriate regions $(x, y, z=$ $-26,-2,-22 ; Z=3.31 ; x, y, z=-38,-46,-18 ; Z=3.81$; $x, y, z=46,-60,-22 ; Z=3.18 ;$ all $P<0.001$ uncorrected) remained, indicating that the effect of high-intensity emotion was emotion-type and task-independent.

\section{Main effect of task}

In the contrast of judging emotion versus gender (i.e., the comparison of direct versus incidental emotional processing, collapsed across emotion subtype and intensity), we observed activation in medial prefrontal cortex/anterior cingulate $(x, y, z=-16,42,-8, Z=5.45)$ (Fig. 6) and posterior cingulate/retrosplenial cortex $(x, y, z=-8,-58,16, Z$ $=5.34$ ) (Fig. 7) (both $P<0.05$ corrected for multiple comparisons). Importantly, however, this latter region was also activated in the interaction between task and intensity (see below). In view of our strong a priori hypotheses, a striking finding was activation in right somatosensory cortex $(x, y, z=44,-12,48, Z=4.02, P<0.05$ SVC) and superior temporal sulcus (STS) $(x, y, z=58,-34,8, Z=$ 3.91, $P<0.05$ SVC) (Fig. 5). Additionally, at uncorrected thresholds of $P<0.001$, activations in predicted regions were evident in bilateral insula $(x, y, z=40,-24,4, Z=$ 4.32; $x, y, z=-40,-16,-8, Z=3.38)$ and SII $(x, y, z=$ $-56,-12,12, Z=3.85)$, although the former failed to correct for multiple comparisons across bilateral insula volume. Conjunction analyses across the distinct emotions for the task effects confirmed that regions highlighted in the main effect of task were activated independent of emotion subtype. In the reverse contrast (judging gender versus judging emotion) there were no significant effects at corrected thresholds.

\section{Interactions between task and intensity}

Only one region reached significance in the contrasts representing the interactions between task and intensity of emotion- posterior rostral motor cingulate $(x, y, z=$ $10,4,48, Z=5.18 ; P<0.05)$. A post hoc test showed that this effect was driven by a greater response to low-intensity faces in the direct condition. Although no other regions reached corrected significance, at an uncorrected threshold of $P<0.001$ we detected voxels in posterior cingulate close to the peaks of activation in the contrast of direct minus incidental task. This suggests that the main effects in this region were partly driven by interactions between intensity of stimulus and task. Post hoc tests revealed that the main effect of task was predominantly a result of a greater re-

Fig. 1. Experimental design and example stimuli. (A) Examples of stimuli used in the experiment. Stimuli were morphs between four male and four female identities from the Ekman series. Morphs were generated between neutral faces of one gender and emotionally expressive faces (disgusted, fearful, happy, or sad) of the other at 10,30, 70, and 90\% along the spectrum between the two starting images. (B) Experimental design: Trials consisted of pairs of faces from the same morph spectrum wherein faces were either 10 and 30\% emotionally expressive (low-intensity trials) or 70 and $90 \%$ emotionally expressive (High-intensity trials). The task was either a judgment of which face was more masculine (incidental trials) or which face was more emotional (direct trials). Faces expressing four different emotions (D, disgust; F, fear; H, happy; S, sad) were used, engendering a $4 \times 2 \times 2$ factorial design. (C) Example series of stimuli and blocking of task. Stimuli were presented as pairs of faces separated by a fixation cross. Task was blocked, each block being preceded by the appropriate task instruction. Blocks consisted of 10 events (stimulus pair or null events). Minimum SOA was $3 \mathrm{~s}$; blocks were $30 \mathrm{~s}$ long.

Fig. 2. Reaction time data across conditions. Mean reaction time for each condition, error bars represent standard error. There is a significant main effect of emotion type, clarified by the interaction between emotion type and task. This effect appears to result from a speeding in the direct task for happy faces and a slowing in the incidental task for fearful faces. 
A

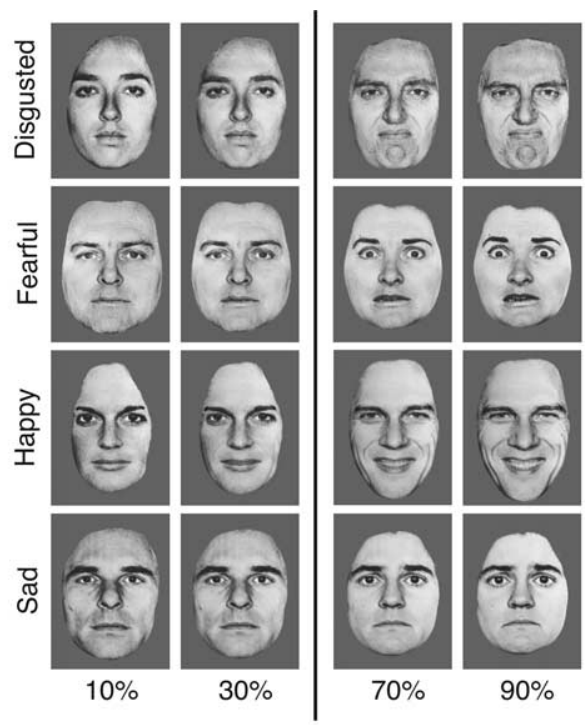

B

Task
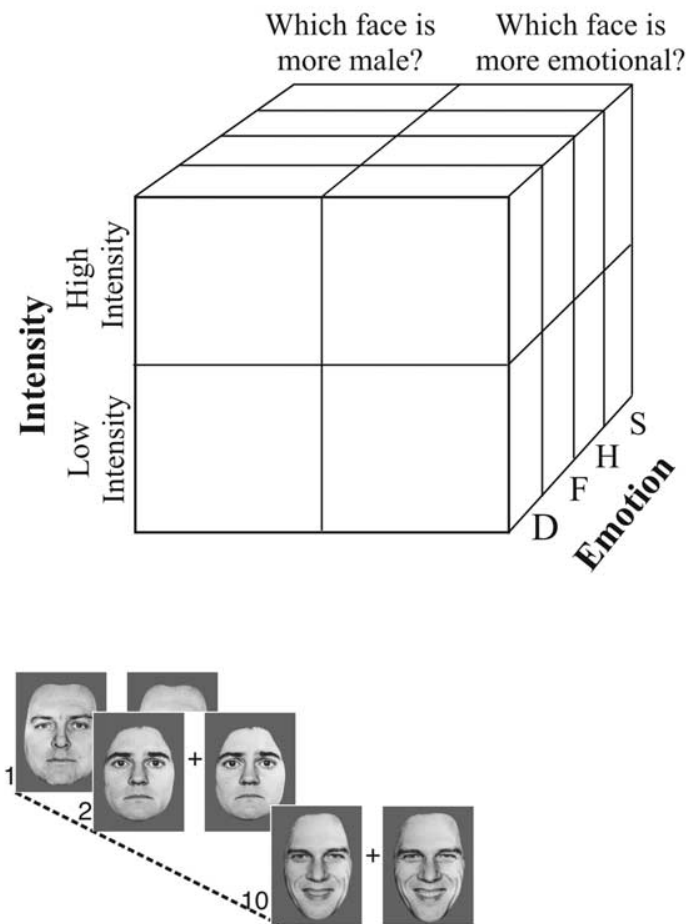

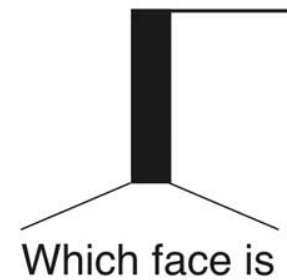

more emotional?
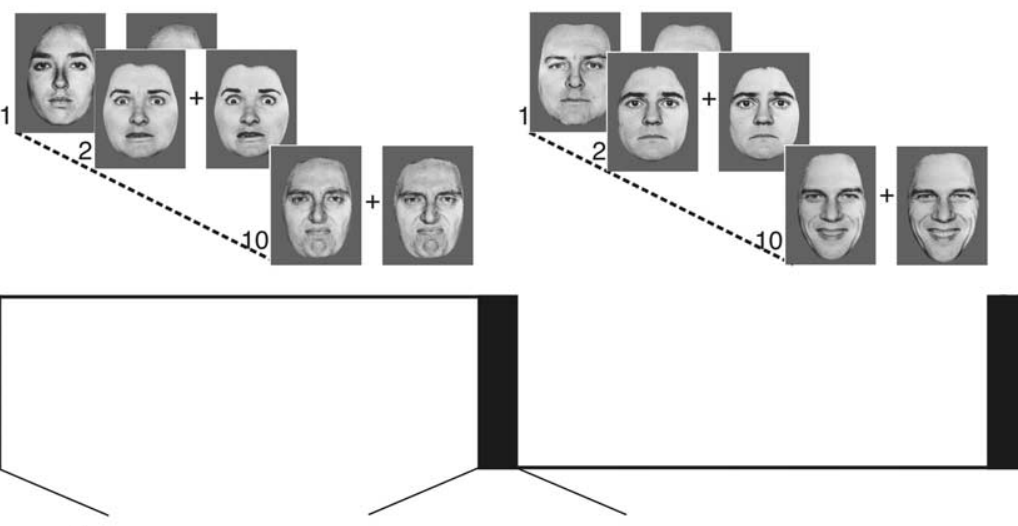

Which face is

more male?
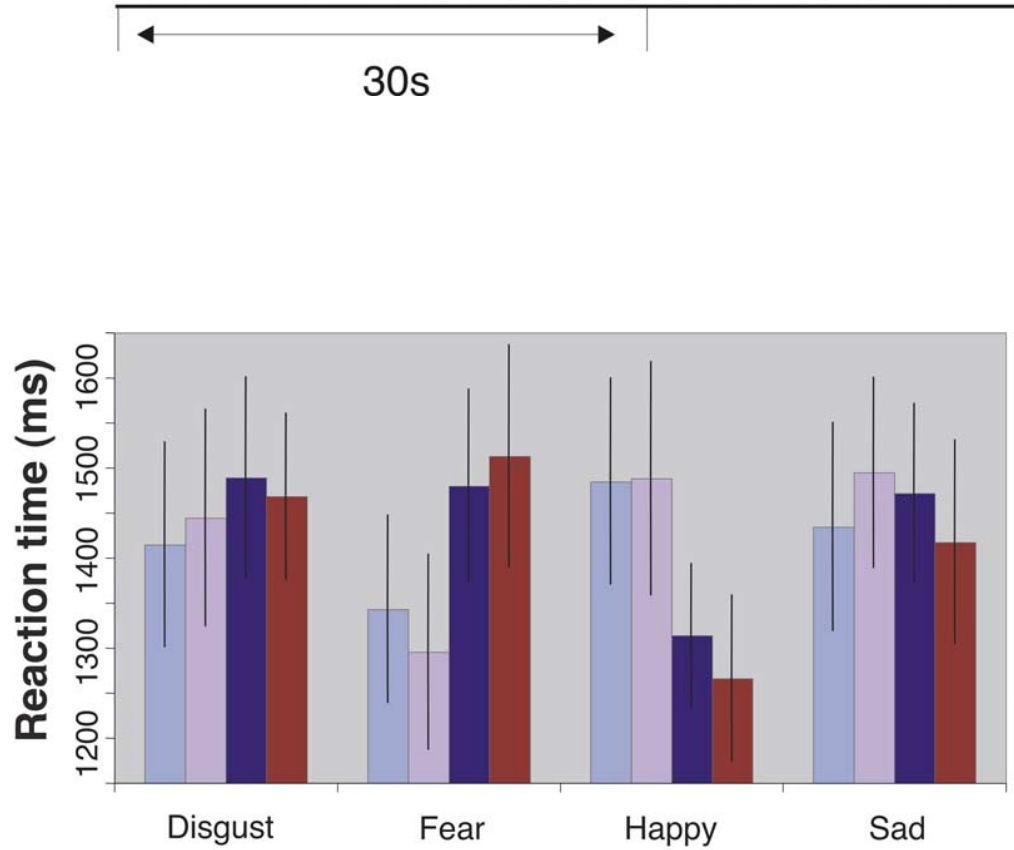

Low intensity,
Incidental Task

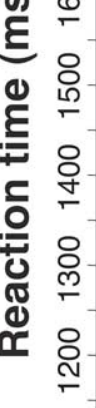

Disgust

Fear

Happy

High intensity,

Incidental Task

Low intensity,

Direct task

High intensity,

Direct task 

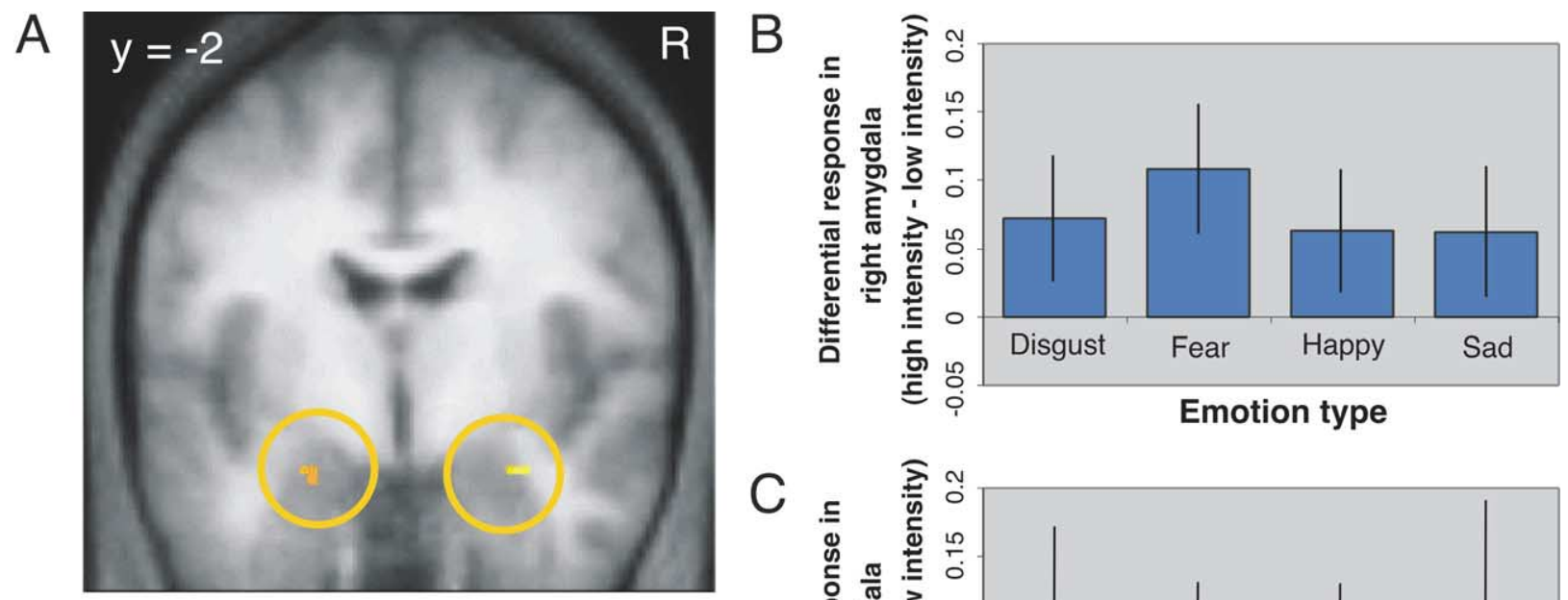

\section{3}
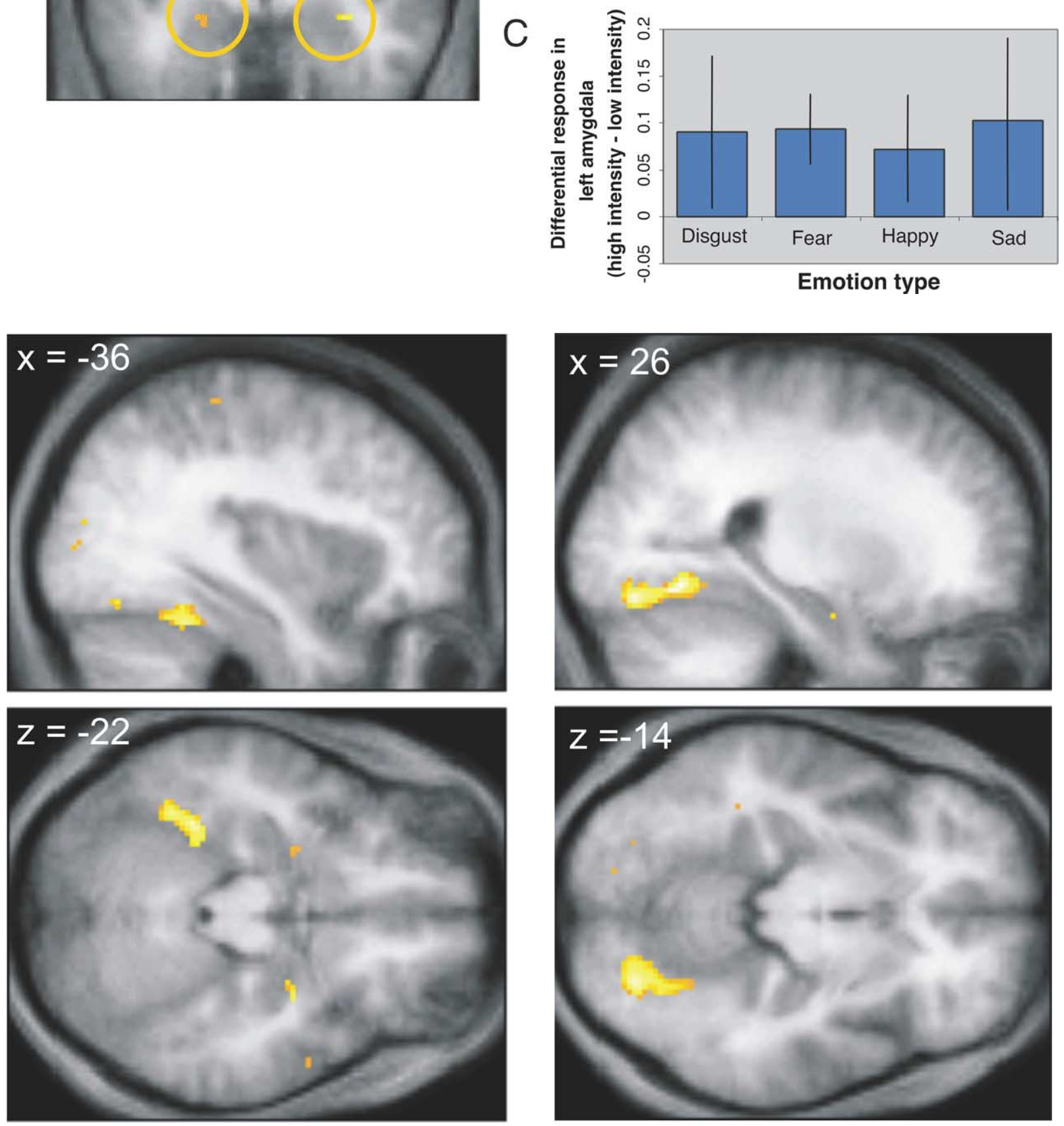

\section{4}
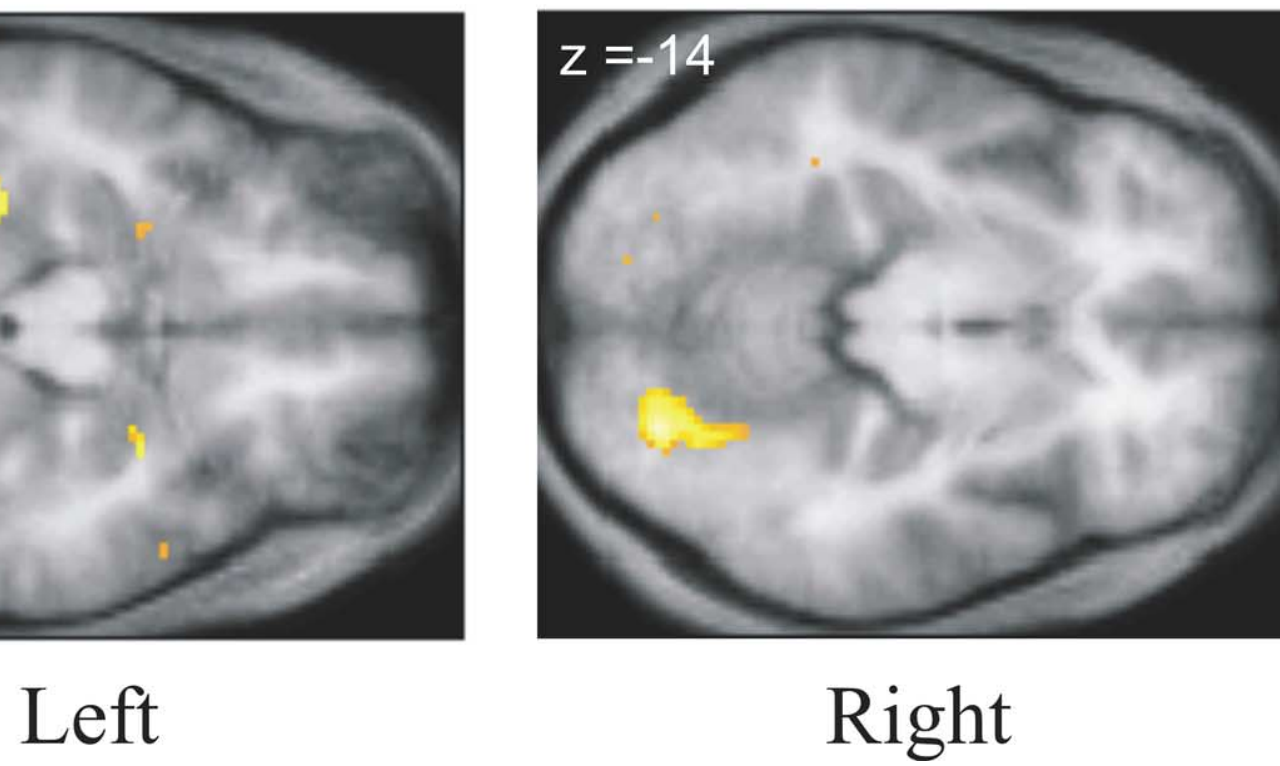

Fig. 3. Amygdala response common to different facial emotions. (A) Bilateral amygdala activation in the conjunction of high intensity-low intensity across emotions (collapsed across task). $\operatorname{SPM}(\mathrm{t})$ thresholded at $P<0.001$ uncorrected overlaid on averaged T1 scan of participants. Right peak at: $x$, $y, z=$ $30,-4,-22 ; Z=3.77$; Left peak at: $x, y, z=-24,-2,-24 ; Z=3.24$ (B) Consistency of effect in right amygdala across emotions. $y$-axis represents size 
sponse to high-intensity emotional faces during the direct task (Fig. 7D).

\section{Differences between emotions}

To examine for brain regions showing differential response to emotion type we used $F$ contrasts in a secondlevel (i.e., random effects) ANOVA model. Distinct contrasts were performed testing for the main effect of emotion, independent of task and intensity, for the interaction between emotion and intensity, for the interaction between emotion and task, and for the three-way interaction. Because we had no specific hypotheses about interactions between emotion and task and the three-way interaction, these latter contrasts were examined only to ensure that in regions reported as significant for the main effects of task and interaction between task and intensity, there was no interaction with emotion type.

No activations survived correction for multiple comparisons in the above contrasts for the differences between emotions. For completeness, we report descriptively activations in predicted regions at $P<0.001$ uncorrected. For the main effect of emotion, activation was seen in posterior right insula $(x, y, z=42,-12,6, Z=3.73)$ where post hoc tests revealed that this effect reflected greater response to disgust faces. The interaction between emotion type and intensity revealed a greater response to high-disgust faces in anterior cingulate $(x, y, z=6,26,24 ; Z=4.53)$ and a decreased response to high-intensity sad faces in anterior insula $(x, y, z=40,18,-14 ; Z=3.83)$. Even at uncorrected thresholds of $P<0.05$, we found no evidence to support the hypothesis of a specific anterior insula response to disgust faces. No regions highlighted in the main effect of task or interaction between task and intensity were shown in the interactions between emotion and task and between emotion, intensity, and task, indicating the independence of the main effects reported in these contrasts from interactions between the different emotions.

Despite the results of the conjunction analysis above (see "Tests for similarities between emotions-conjunction analysis") highlighting a common response within amygdala to high-intensity expressions of the four emotions, there remains the possibility of an interaction between emotion type and intensity. For example, a high-intensity expression of one emotion type might activate the amygdala to a greater degree than high-intensity exemplars of other emotions, even though both activate amygdala relative to their lowintensity counterparts. As the existence of such interactions might critically influence the interpretation of our results, we investigated such interactions at lowered statistical thresholds within the amygdala. Even at thresholds of $P<$ 0.1 uncorrected there was no evidence for fear-selectivity in voxels within amygdala. Indeed, at such low thresholds, where false positives are poorly protected against, the voxels which showed interactions by emotion type appeared more responsive to disgust or combinations of disgust and sadness than to fear.

\section{Discussion}

The goal of this study was to address the question whether task engenders differential neural responses to the perception of facial expressions of emotion, and to what degree these neural responses are common to distinct categories of emotions. By using a multifactorial event-related fMRI design which required either direct or incidental processing of four different facial emotions we indexed the task dependence of neural responses to these emotions as well as their specificity to one or more emotion type. A critical finding is our demonstration of task-independent amygdala responses in a conjunction of high-intensity minus lowintensity expressions across four basic emotions. Thus, the null hypothesis that at least one of these emotions failed to activate the amygdala can be rejected. The findings that the conjunction of high-intensity minus low-intensity across task and emotion yields amygdala activation, and an absence of interaction between intensity and task in the amygdala, provide evidence that this region responds to emotionally salient stimuli independent of task. This finding is consistent with previous indications that the amygdala shows a high degree of automaticity in responding to emotive stimuli, independent of task, attention, or awareness (Dolan, 2002; Morris et al., 1998b; Ohman, 2002; Vuilleumier et al., 2001; Whalen et al., 1998; Winston et al., 2002).

Although the neuroimaging and neuropsychological literature on the topic of perception of facial expressions of emotion has tended to emphasise roles for the amygdala in perception of negative facial emotions (Adolphs et al., 1999; Adolphs, 2002b; Morris et al., 1996), a compelling animal and human literature implicates the amygdala in positive affective learning and perception (Baxter and Murray, 2002; Garavan et al., 2001; Gottfried et al., 2002a,b; Johnsrude et al., 2000; O'Doherty et al., 2001; Ono and Nishijo, 1999; Parkinson et al., 2001; Rolls, 1999). A more considered view, therefore, might be to suggest that the

of differential response to high- and low-intensity expressions in amygdala (arbitrary units). Note that although there is a trend to greater response to fear than to the other emotions, this trend is absent in other voxels within amygdala (e.g., (C) and does not attain statistical significance $(P>0.8)$. Importantly, if fear is omitted from the conjunction, amygdala voxels still obtain from this analysis. (C) High- versus low-intensity expressions for individual emotions in left amygdala.

Fig. 4. Fusiform and extrastriate activations common to distinct facial emotions. Bilateral fusiform and extrastriate cortex activation in the conjunction of high-intensity minus low-intensity emotion expression across emotions (collapsed across task). Right peak at: $x, y, z=28,-66,-12 ; Z=4.57$; Left peak at: $x, y, z=-30,-42,-20 ; Z=4.26$ Display as in Fig. 3A. 
amygdala responds to stimuli of motivational significance, independent of their emotional valence.

It is important to consider why previous imaging studies concerning perception of facial expressions have reported different results, particularly with regard to the role of the amygdala. The majority of previous studies have adopted block designs, which have two major disadvantages. The first is the inability of blocked designs to separate effects due to anticipation versus processing of a given stimulus. The second is the possibility of differential habituation in emotion processing regions to repeated presentations of different emotions, an effect previously reported (Breiter et al., 1996; Wright et al., 2001). Randomized event-related designs, as adopted here, are less susceptible to habituation effects. Thus, in previous block designs, amygdala responses may have differed between different emotions due to a role for this region in anticipation of a given emotional stimulus, or more rapid adaptation to one stimulus type than another. Either of these effects might account for apparent discrepancy between the results of our study and those already in the literature (e.g., Morris et al., 1996; Phillips et al., 1997, 1998), particularly with respect to the amygdala. Indeed, it should be noted that although our positive finding from a conjunction analysis allows the rejection of the null hypothesis that at least one of the high-intensity expressions failed to activate amygdala more than its low-intensity counterpart, we cannot conclude that no differences exist in the amygdala between the processing of the distinct emotions. To draw such an inference would be to accept the null hypothesis tested under the tests for differences between the emotions. We consider it noteworthy, however, that regions other than the amygdala (e.g., anterior cingulate and posterior insula) did show selective responses, which indicates that our experimental design had sufficient power to detect differential responses. Of course, it is possible that more events per condition or a higher field strength scanner might prove more sensitive to differences between conditions in this region. Although the peak voxel in right amygdala shows a trend toward greater responses to fearful faces (Fig. $3 \mathrm{~B})$, this trend does not even approach significance $(P>$ 0.8 ). Other voxels within the amygdala region highlighted by the conjunction do not show such a trend (e.g., left amygdala peak in Fig. 3C). As noted in the Introduction, the findings from human patients with lesions in the region of the amygdala are less consistent than assumed. A recent meta-analysis (Fine and Blair, 2000) highlights the fact that only two patients with fear-selective deficits (of a total of 13) have been reported, and one other patient (EP) showed an anger-selective impairment. Although it is apparent that fear recognition is the most common impairment in this patient group, it is argued that fear is the hardest emotion for healthy subjects to recognize, and the association of fear recognition deficits and amygdala damage may partly reflect task difficulty effects (Rapcsak et al., 2000). Indeed, when the performance of neurologically intact subjects on the specific emotions is factored into an analysis of amygdala- damaged patients, no fear-specific deficit is found (Rapcsak et al., 2000). It is interesting to note that single neuron recordings in human amygdala have failed to show a general selectivity for fear, instead finding single neurons with responses to single emotions, but no prevalence of one emotion type (Fried et al., 1997).

A contrast between the direct and incidental task revealed significant differential activity in rostral anterior cingulate and ventromedial prefrontal cortex, indicating that these regions mediate explicit representation of emotions. We note that ventral frontal cortex has previously been implicated in emotion recognition in faces and other modalities in neuropsychological studies (Hornak et al., 1996). The activation we report accords with a proposed role for ventromedial prefrontal cortex in intentional expression of emotional and social behavior (Anderson et al., 1999; Dimitrov et al., 1999; Eslinger, 1998; Eslinger and Damasio, 1985; Grattan and Eslinger, 1992). A mechanism for recognition of a conspecific's emotional states has been proposed to involve a form of simulation within one's own cortical circuitry (Adolphs et al., 2000; Adolphs, 2002a, $2002 \mathrm{~b}$ ). In this regard it is noteworthy that anterior cingulate/ventromedial prefrontal cortex is involved in generation and reafferent mapping of bodily states (Critchley et al., 2000b) that constitute an integral part of emotional experience (Damasio, 1994; James, 1884). There is also evidence for enhanced activation in this region of ventromedial prefrontal cortex when subjects attend to their subjective emotional response to stimuli (Lane et al., 1997).

A striking finding was enhanced activation in somatosensory cortex and insula during the direct task, although the latter failed to correct for multiple comparisons across bilateral insula volume. Activations in these regions accord with the proposal that recognition of emotion involves simulation of the emotional display of a conspecific (Adolphs et al., 2000). However, it is interesting that we reveal activation in these regions in a main effect of task, and not in a main effect of intensity. The latter would be more compatible with a model in which simple perception of emotional faces entailed activation in these regions. The fact that the former is the case suggests that patients' deficits in emotion recognition tasks following lesions in ventral prefrontal and right somatosensory regions may reflect impairment in deliberative categorizing of facial expressions despite intact basic perception. Activation in motor cingulate in the interaction between task and intensity of emotion, driven by an increased response to low-intensity expressions during the direct task, might reflect greater effort for this condition. One possibility is that low-intensity expressions required increased processing in the service of successful recognition.

The possible role of retrosplenial cortex in human emotional function has been highlighted by the suggestion that this region is commonly activated in neuroimaging studies of emotion (Maddock, 1999). This region is also strongly implicated in episodic memory retrieval processes (Fink et 
A

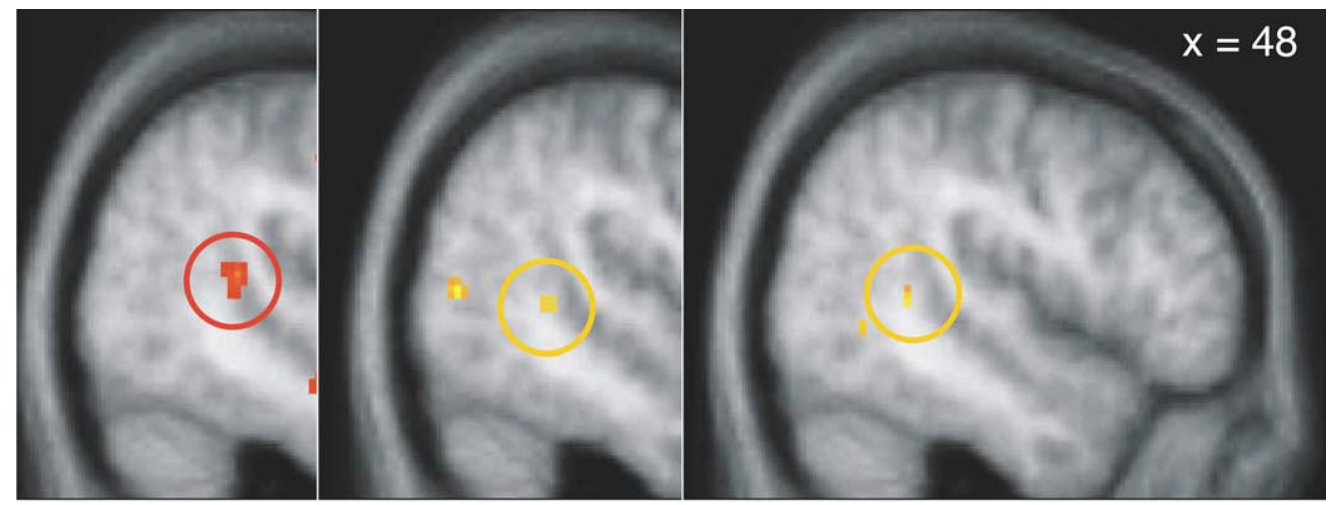

Direct - High intensity incidental low intensity

High intensity low intensity (conjunction)

B

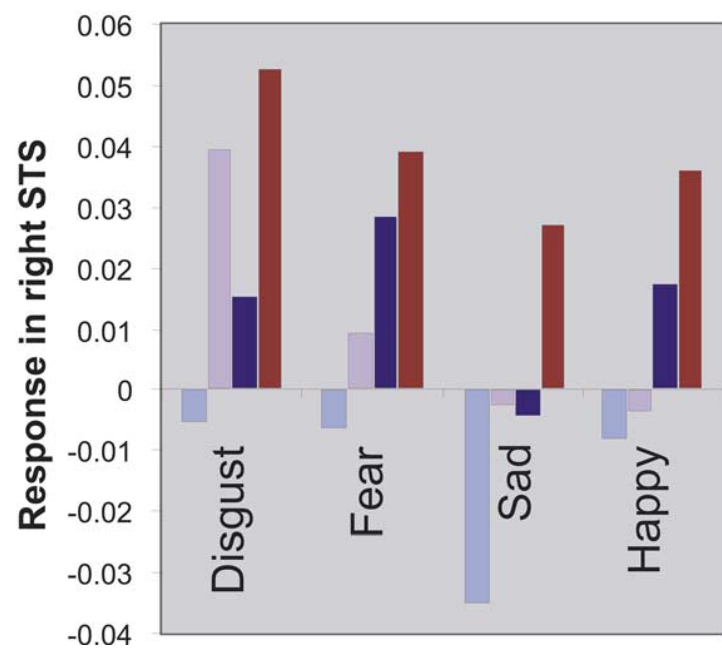

Low intensity,

Incidental Task

High intensity,

Incidental Task

Low intensity,

Direct task

High intensity, Direct task
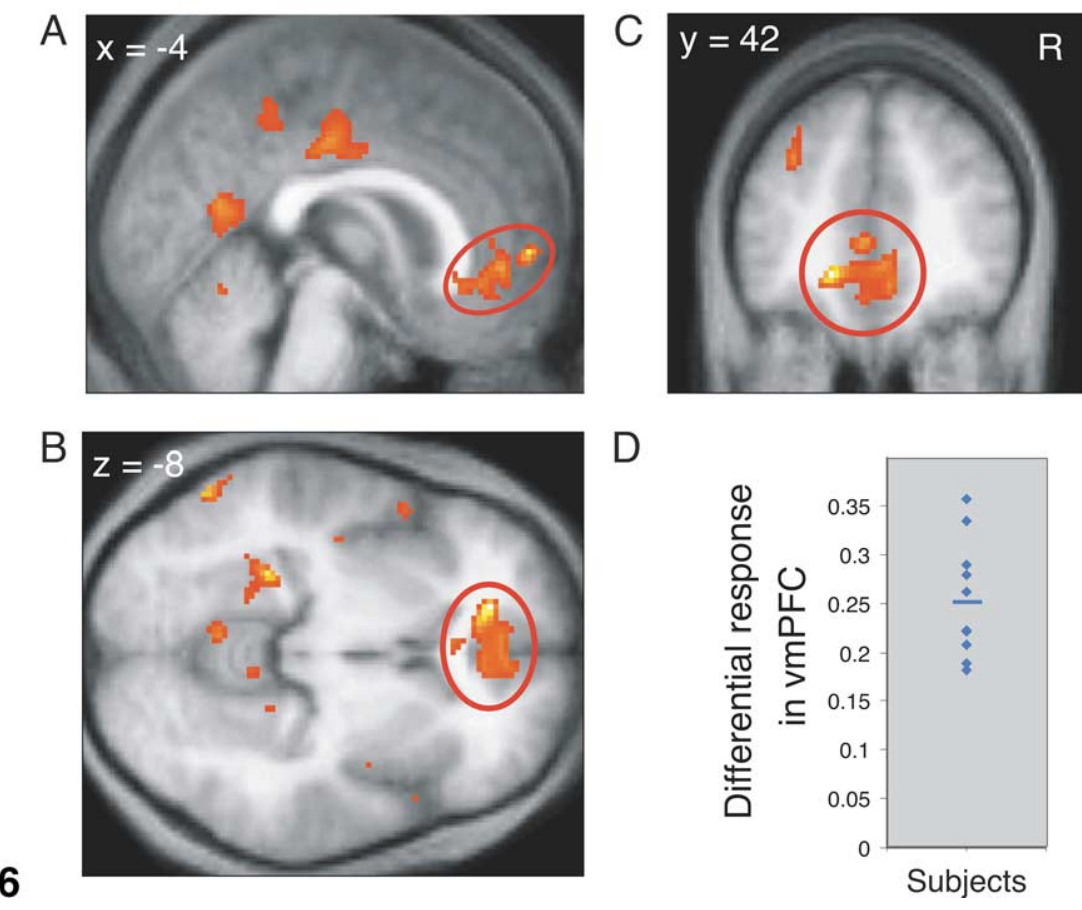

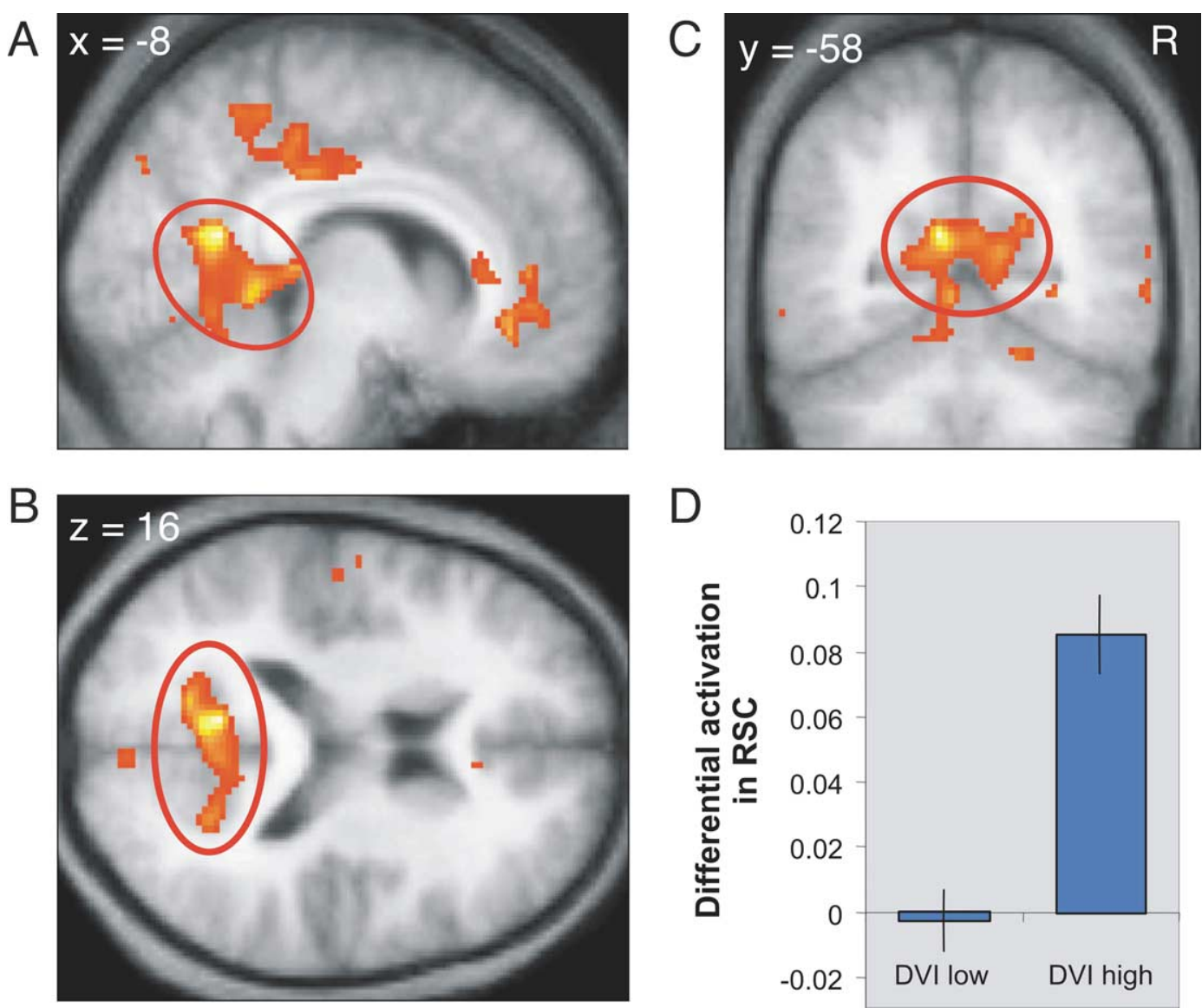

Fig. 7. Retrosplenial cortex shows interaction between task and intensity. (A-C) Sagittal, horizontal, and coronal sections showing significant response during direct task compared to incidental task in retrosplenial/posterior cingulate cortex (RSC). However, this region was also highlighted as showing a significant interaction between task and intensity. Display as in Fig. 3A. (D) Demonstration that main effect of task is a result of greater responses to high-intensity expression faces during the direct task than the incidental task. $y$-axis represents size of differential response in peak voxel (arbitrary units). DVI low, direct versus incidental task for low intensity expressions only; DVI high, direct versus incidental task for high intensity expressions only.

al., 1996; Maguire, 2001a, 2001b). Even within the context of a role for this region in mediating familiarity, a recent study observed a trend suggestive of a specialization for face stimuli over voice stimuli (Shah et al., 2001). In our study, this region was activated by the main effect of task (direct versus incidental) and in the interaction between task and emotion intensity resulting from enhanced activity during presentation of high-intensity faces specific to the direct task (Fig. 7). Although a prediction from the literature on this region's responses to emotional stimuli would be a main effect of intensity, our data suggest that it responds to emotionally salient stimuli under explicit conditions. Indeed, we note that five of the six "well-controlled studies" highlighted by a meta-analysis of functional imaging studies of emotion as showing activation in this region (Maddock, 1999) utilized tasks requiring explicit evaluation or recall of stimuli or situations. Thus, we propose that retrosplenial cortex is involved in direct evaluation of highly emotive stimuli which may reflect a necessity to recall stored representations of emotional information.

Both fusiform cortex and posterior STS exhibit faceresponsiveness as measured by fMRI (Halgren et al., 1999; Hoffman and Haxby, 2000; Kanwisher et al., 1997; KeslerWest et al., 2001). A recently proposed neuroanatomical model of face perception highlighted dissociable roles of these two regions (Haxby et al., 2000). The model proposes

Fig. 5. Responses in right posterior superior temporal sulcus (STS). (A) Response in right posterior STS to task (direct $>$ incidental), intensity (high $>$ low), and conjunction of intensity across emotions. Display as in Fig. 3A. (B) Response to individual event types in right STS. Note the increased response to high-intensity expressions for each emotion and the additive effect of task, wherein responses are greater in the direct task. $y$-axis represents size of effect in peak voxel (arbitrary units).

Fig. 6. Ventromedial prefrontal cortex responses in direct task. (A-C) Sagittal, horizontal, and coronal sections showing highly significant response during direct task compared to incidental task in ventromedial prefrontal cortex (vmPFC). Display as in Fig. 3A. (D) Consistency of response in peak voxel in vmPFC. Diamonds represent individual subjects; horizontal line, the mean response. $y$-axis represents size of differential response in vmPFC (arbitrary units). 
that fusiform cortex is part of a core system for visual analysis of faces that responds to invariant aspects of faces such as identity, whereas STS codes changeable aspects of faces, including eye gaze and facial expression. Our data broadly conform to this model. We also demonstrate increased fusiform responses to emotional faces, which might be attributed to greater processing resources being deployed to emotionally salient stimuli (Dolan, 2002), effects that might relate to feedback from amygdala to visual areas (Amaral et al., 1992; Morris et al., 1998a). In STS, although the same general pattern of enhanced BOLD responses to emotional faces is seen, it seems probable on the basis of single unit studies in non-human primates (Hasselmo et al., 1989) that this reflects specific coding for facial expressions within this region, rather than a more general effect of enhanced attentional processing. An additional dissociation between these two regions is the task-dependent nature of the response in STS that took the form of an additive effect of high-intensity expressions and direct task, in the absence of an interaction. Attention to facial emotion, or even to emotive facial characteristics such as trustworthiness, activates STS (Narumoto et al., 2001; Streit et al., 1999; Winston et al., 2002). One possibility is that our direct task engenders the sort of "intention detection" that has been hypothesized to invoke this region (Frith and Frith, 1999; Jellema et al., 2000).

A current trend in the literature is that of highlighting dissociations between neural substrates for perception of different emotions (Phillips et al., 1998; Calder et al., 2001). In this context we consider the paucity of regions displaying such dissociations as of interest. Although some differences were apparent at uncorrected thresholds in "limbic" areas (insula and anterior cingulate) these differences did not accord with our predictions from the literature. For example, increased activation to disgust would be predicted in anterior insula (Calder et al., 2001), and although some differences were evident in this region, they were not a result of selective responses to disgust faces. We do not associate the lack of evidence for differences between emotions in this study as supportive of dimensional accounts of emotion. Indeed, a simple dimensional account of emotion would not predict that a single region responds to highintensity exemplars of emotions that represent the opposite ends of the valence (e.g., happy and disgusted) and intensity (e.g., fearful and sad) spectra. Rather, we are persuaded by the behavioral evidence for categorical emotion perception (e.g., Etcoff and Magee, 1992; Young et al., 1996) but suspect that coding for the different emotions may exist on a spatial scale that borders the limits of our current technique. This could conceivably take the form of single neurons coding for distinct emotions within the same brain region (e.g., STS).

In conclusion, our data indicate a common neural substrate for perception of different facial emotions involving the amygdala, extrastriate and fusiform cortex, and posterior STS. Taken in conjunction with our failure to demonstrate regions exhibiting convincing differences between the four different emotions studied here, these data challenge the concept of functional segregation for perception of individual emotions at a gross anatomical level. Increased responses to highly emotional faces in amygdala and fusiform were task-independent, concomitant with a hypothesis of obligatory processing of emotion in these regions. Taskdependent increases in neural responses in ventromedial prefrontal cortex and somatosensory regions suggest that the impairment in emotion recognition of patients with lesions in these regions is a function of lack of integration between intact perceptual systems and systems that provide "somatic" cues that can bias overt emotional judgments.

\section{Acknowledgments}

We thank the radiographers at the FIL for assistance with scanning; D. Perrett for the masked Ekman stimuli; and K. Friston, R. Henson, W. Penny, D. Glaser, E. Maguire, P. Bentley, and J. Gottfried for helpful discussions. This work was supported by a programme grant to R.J.D. from the Wellcome Trust.

\section{References}

Adolphs, R., 1999. Social cognition and the human brain. Trends Cogn. Sci. 3, 469-479.

Adolphs, R., 2002a. Neural systems for recognizing emotion. Curr. Opin. Neurobiol. 12, 169-177.

Adolphs, R., 2002b. Recognizing emotion from facial expressions: psychological and neurological mechanisms. Behavior. Cogn. Neurosci. Rev. 1, 21-61.

Adolphs, R., Damasio, H., Tranel, D., Cooper, G., Damasio, A.R., 2000. A role for somatosensory cortices in the visual recognition of emotion as revealed by three-dimensional lesion mapping. J. Neurosci. 20, $2683-$ 2690.

Adolphs, R., Damasio, H., Tranel, D., Damasio, A.R., 1996. Cortical systems for the recognition of emotion in facial expressions. J. Neurosci. 16, 7678-7687.

Adolphs, R., Tranel, D., Damasio, H., Damasio, A., 1994. Impaired recognition of emotion in facial expressions following bilateral damage to the human amygdala. Nature 372, 669-672.

Adolphs, R., Tranel, D., Damasio, H., Damasio, A.R., 1995. Fear and the human amygdala. J. Neurosci. 15, 5879-5891.

Adolphs, R., Tranel, D., Hamann, S., Young, A.W., Calder, A.J., Phelps, E.A., Anderson, A., Lee, G.P., Damasio, A.R., 1999. Recognition of facial emotion in nine individuals with bilateral amygdala damage. Neuropsychologia 37, 1111-1117.

Amaral, D.G., Price, J.L., Pitkänen, A., Carmichael, S.T., 1992. Anatomical organization of the primate amygdaloid complex, in: Aggleton, J.P. (Ed.), The Amygdala: Neurobiological Aspects of Emotion, Memory, and Mental Dysfunction. Wiley-Liss, New York, pp. 1-66.

Anderson, S.W., Bechara, A., Damasio, H., Tranel, D., Damasio, A.R., 1999. Impairment of social and moral behavior related to early damage in human prefrontal cortex. Nat. Neurosci. 2, 1032-1037.

Baxter, M.G., Murray, E.A., 2002. The amygdala and reward. Nat. Rev. Neurosci. 3, 563-573.

Blair, R.J., Morris, J.S., Frith, C.D., Perrett, D.I., Dolan, R.J., 1999. Dissociable neural responses to facial expressions of sadness and anger. Brain 122, 883-893. 
Breiter, H.C., Etcoff, N.L., Whalen, P.J., Kennedy, W.A., Rauch, S.L., Buckner, R.L., Strauss, M.M., Hyman, S.E., Rosen, B.R., 1996. Response and habituation of the human amygdala during visual processing of facial expression. Neuron 17, 875-887.

Broks, P., Young, A.W., Maratos, E.J., Coffey, P.J., Calder, A.J., Isaac, C.L., Mayes, A.R., Hodges, J.R., Montaldi, D., Cezayirli, E., Roberts, N., Hadley, D., 1998. Face processing impairments after encephalitis: amygdala damage and recognition of fear. Neuropsychologia 36, 5970.

Brothers, L., 1990. The social brain: a project for integrating primate behavior and neurophysiology in a new domain. Concepts Neurosci. 1, 27-51.

Buchel, C., Dolan, R.J., Armony, J.L., Friston, K.J., 1999. Amygdalahippocampal involvement in human aversive trace conditioning revealed through event-related functional magnetic resonance imaging. J. Neurosci. 19, 10869-10876.

Buchel, C., Morris, J., Dolan, R.J., Friston, K.J., 1998. Brain systems mediating aversive conditioning: an event-related fMRI study. Neuron 20, 947-957.

Calder, A.J., Keane, J., Manes, F., Antoun, N., Young, A.W., 2000. Impaired recognition and experience of disgust following brain injury. Nat. Neurosci. 3, 1077-1078.

Calder, A.J., Lawrence, A.D., Young, A.W., 2001. Neuropsychology of fear and loathing. Nat. Rev. Neurosci. 2, 352-363.

Calder, A.J., Young, A.W., Rowland, D., Perrett, D.I., Hodges, J.R., Etcoff, N.L., 1996. Facial emotion recognition after bilateral amygdala damage-differentially severe impairment of fear. Cogn. Neuropsychol. 13, 699-745.

Collignon, A., Maes, F., Delaere, D., Vandermeulen, D., Suetens, P., Marchal, G., 1995. Automated multi-modality image registration based on information theory, in: Bizais, Y., Barillot, C., Di Paola, R. (Eds.), Proc. Information Processing in Medical Imaging 1995. Kluwer Academic Publishers, Dordrecht, The Netherlands, pp. 263-274.

Critchley, H., Daly, E., Phillips, M., Brammer, M., Bullmore, E., Williams, S., Van Amelsvoort, T., Robertson, D., David, A., Murphy, D., 2000a. Explicit and implicit neural mechanisms for processing of social information from facial expressions: a functional magnetic resonance imaging study. Human Brain Mapping 9, 93-105.

Critchley, H.D., Elliott, R., Mathias, C.J., Dolan, R.J., 2000b. Neural activity relating to generation and representation of galvanic skin conductance responses: a functional magnetic resonance imaging study. J. Neurosci. 20, 3033-3040.

Damasio, A.R., 1994. Descartes' Error: Emotion, Reason and the Human Brain. G.P. Putnam's Sons, New York.

Damasio, A., 1999. The Feeling of What Happens: Body and Emotion in the Making of Consciousness. Harcourt Brace, New York.

Darwin, C.R., 1872. The Expression of the Emotions in Man and Animals John Murray, London.

Dimitrov, M., Phipps, M., Zahn, T.P., Grafman, J., 1999. A thoroughly modern gage. Neurocase 5, 345-353.

Dolan, R.J., 2002. Emotion, cognition, and behavior. Science 298, 11911194.

Dolan, R.J., Fletcher, P., Morris, J., Kapur, N., Deakin, J.F., Frith, C.D., 1996. Neural activation during covert processing of positive emotional facial expressions. Neuroimage 4, 194-200.

Dolan, R.J., Morris, J.S., de Gelder, B., 2001. Crossmodal binding of fear in voice and face. Proc. Natl. Acad. Sci. USA 98, 10006-10010.

Ekman, P., 1993. Facial expression and emotion. Am. Psychol. 48, $384-$ 392.

Ekman, P., Friesen, W.V., 1976. Pictures of Facial Affect. Consulting Psychologists Press, Palo Alto, CA.

Eslinger, P.J., 1998. Neurological and neuropsychological bases of empathy. Eur. Neurol. 39, 193-199.

Eslinger, P.J., Damasio, A.R., 1985. Severe disturbance of higher cognition after bilateral frontal lobe ablation: patient EVR. Neurology 35, 17311741.
Etcoff, N.L., Magee, J.J., 1992. Categorical perception of facial expressions. Cognition 44, 227-240.

Fine, C., Blair, R.J., 2000. The cognitive and emotional effects of amygdala damage. Neurocase 6, 435-438.

Fink, G.R., Markowitsch, H.J., Reinkemeier, M., Bruckbauer, T., Kessler, J., Heiss, W.D., 1996. Cerebral representation of one's own past: neural networks involved in autobiographical memory. J. Neurosci. 16, 42754282.

Fried, I., MacDonald, K.A., Wilson, C.L., 1997. Single neuron activity in human hippocampus and amygdala during recognition of faces and objects. Neuron 18, 753-765.

Friston, K., Ashburner, J., Frith, C.D., Poline, J.-B., Heather, J.D., Frackowiak, R.S.J., 1995a. Spatial registration and normalization of images. Human Brain Mapping 2, 165-189.

Friston, K., Holmes, A.P., Worsley, K., Poline, J.-B., Frith, C., Frackowiak, R.S.J., 1995b. Statistical parametric maps in functional imaging: a general linear approach. Human Brain Mapping 2, 189-210.

Friston, K.J., Fletcher, P., Josephs, O., Holmes, A., Rugg, M.D., Turner, R., 1998. Event-related fMRI: characterizing differential responses. Neuroimage 7, 30-40.

Friston, K.J., Holmes, A.P., Worsley, K.J., 1999. How many subjects constitute a study? Neuroimage 10, 1-5.

Frith, C.D., Frith, U., 1999. Interacting minds-a biological basis. Science 286, 1692-1695.

Garavan, H., Pendergrass, J.C., Ross, T.J., Stein, E.A., Risinger, R.C., 2001. Amygdala response to both positively and negatively valenced stimuli. NeuroReport 12, 2779-2783.

Glaser, D., Penny, W., Henson, R., Friston, K., 2002. Coping with the variance structure of neuroimaging data. J. Cogn. Neurosci. Supplement S, E92 [abstract].

Gorno-Tempini, M.L., Pradelli, S., Serafini, M., Pagnoni, G., Baraldi, P., Porro, C., Nicoletti, R., Umita, C., Nichelli, P., 2001. Explicit and incidental facial expression processing: an fMRI study. Neuroimage $14,465-473$

Gottfried, J.A., Deichmann, R., Winston, J.S., Dolan, R.J., 2002a. Functional heterogeneity in human olfactory cortex: an event-related functional magnetic resonance imaging study. J. Neurosci. 22, 1081910828.

Gottfried, J.A., O’Doherty, J., Dolan, R.J., 2002b. Appetitive and aversive olfactory learning in humans studied using event-related functional magnetic resonance imaging. J. Neurosci. 22, 10829-10837.

Grattan, L.M., Eslinger, P.J., 1992. Long-term psychological consequences of childhood frontal lobe lesion in patient DT. Brain Cogn. 20, 185195.

Halgren, E., Dale, A.M., Sereno, M.I., Tootell, R.B., Marinkovic, K., Rosen, B.R., 1999. Location of human face-selective cortex with respect to retinotopic areas. Human Brain Mapping 7, 29-37.

Hamann, S.B., Stefanacci, L., Squire, L.R., Adolphs, R., Tranel, D., Damasio, H., Damasio, A., 1996. Recognizing facial emotion. Nature 379, 497

Hariri, A.R., Bookheimer, S.Y., Mazziotta, J.C., 2000. Modulating emotional responses: effects of a neocortical network on the limbic system. NeuroReport 11, 43-48.

Hasselmo, M.E., Rolls, E.T., Baylis, G.C., 1989. The role of expression and identity in the face-selective responses of neurons in the temporal visual cortex of the monkey. Behav. Brain Res. 32, 203-218.

Haxby, J.V., Hoffman, E.A., Gobbini, M.I., 2000. The distributed human neural system for face perception. Trends Cogn. Sci. 4, 223-233.

Haxby, J.V., Ungerleider, L.G., Clark, V.P., Schouten, J.L., Hoffman, E.A., Martin, A., 1999. The effect of face inversion on activity in human neural systems for face and object perception. Neuron 22, 189-199.

Henson, R.N.A., Buechel, C., Josephs, D., Friston, K., 1999. The slicetiming problem in event-related fMRI. NeuroImage 9, 125 [abstract].

Hoffman, E.A., Haxby, J.V., 2000. Distinct representations of eye gaze and identity in the distributed human neural system for face perception. Nat. Neurosci. 3, 80-84. 
Hornak, J., Rolls, E.T., Wade, D., 1996. Face and voice expression identification in patients with emotional and behavioural changes following ventral frontal lobe damage. Neuropsychologia 34, 247-261.

James, W., 1884. What is an emotion? Mind 9, 188-205.

Jellema, T., Baker, C.I., Wicker, B., Perrett, D.I., 2000. Neural representation for the perception of the intentionality of actions. Brain Cogn. 44, 280-302.

Johnsrude, I.S., Owen, A.M., White, N.M., Zhao, W.V., Bohbot, V., 2000. Impaired preference conditioning after anterior temporal lobe resection in humans. J. Neurosci. 20, 2649-2656.

Kanwisher, N., McDermott, J., Chun, M.M., 1997. The fusiform face area a module in human extrastriate cortex specialized for face perception. J. Neurosci. 17, 4302-4311.

Kesler-West, M.L., Andersen, A.H., Smith, C.D., Avison, M.J., Davis, C.E., Kryscio, R.J., Blonder, L.X., 2001. Neural substrates of facial emotion processing using fMRI. Brain Res. Cogn. Brain Res. 11, 213-226.

Kolb, B., Milner, B., Taylor, L., 1983. Perception of faces by patients with localized cortical excisions. Can. J. Psychol. 37, 8-18.

Lane, R.D., Fink, G.R., Chau, P.M., Dolan, R.J., 1997. Neural activation during selective attention to subjective emotional responses. NeuroReport 8, 3969-3972.

Maddock, R.J., 1999. The retrosplenial cortex and emotion: new insights from functional neuroimaging of the human brain. Trends Neurosci. 22, 310-316.

Maguire, E.A., 2001a. Neuroimaging studies of autobiographical event memory. Philos. Trans. R. Soc. London B Biol. Sci. 356, 1441-1451.

Maguire, E.A., 2001b. The retrosplenial contribution to human navigation: a review of lesion and neuroimaging findings. Scand. J. Psychol. 42, 225-238.

Morris, J.S., Friston, K.J., Buchel, C., Frith, C.D., Young, A.W., Calder, A.J., Dolan, R.J., 1998a. A neuromodulatory role for the human amygdala in processing emotional facial expressions. Brain 121, 47-57.

Morris, J.S., Frith, C.D., Perrett, D.I., Rowland, D., Young, A.W., Calder, A.J., Dolan, R.J., 1996. A differential neural response in the human amygdala to fearful and happy facial expressions. Nature 383, 812815.

Morris, J.S., Ohman, A., Dolan, R.J., 1998b. Conscious and unconscious emotional learning in the human amygdala. Nature 393, 467-470.

Narumoto, J., Okada, T., Sadato, N., Fukui, K., Yonekura, Y., 2001. Attention to emotion modulates fMRI activity in human right superior temporal sulcus. Brain Res. Cogn. Brain Res. 121, 225-231.

O’Doherty, J., Rolls, E.T., Francis, S., Bowtell, R., McGlone, F., 2001 Representation of pleasant and aversive taste in the human brain. J. Neurophysiol. 85, 1315-1321.

Ohman, A., 2002. Automaticity and the amygdala: nonconscious responses to emotional faces. Curr. Direct. Psychologic. Sci. 11, 62-66.

Ono, T., Nishijo, H., 1999. Neurophysiological basis of emotion in primates: neuronal responses in the monkey amygdala and anterior cingulate cortex, in: Gazzaniga, M.S. (Ed.), The New Cognitive Neurosciences. MIT Press, Cambridge, MA, pp. 1099-1114.

Parkinson, J.A., Crofts, H.S., McGuigan, M., Tomic, D.L., Everitt, B.J., Roberts, A.C., 2001. The role of the primate amygdala in conditioned reinforcement. J. Neurosci. 21, 7770-7780.

Phillips, M.L., Medford, N., Young, A.W., Williams, L., Williams, S.C., Bullmore, E.T., Gray, J.A., Brammer, M.J., 2001. Time courses of left and right amygdalar responses to fearful facial expressions. Human Brain Mapping 12, 193-202.

Phillips, M.L., Williams, L., Senior, C., Bullmore, E.T., Brammer, M.J., Andrew, C., Williams, S.C., David, A.S., 1999. A differential neural response to threatening and non-threatening negative facial expressions in paranoid and non-paranoid schizophrenics. Psychiatry Res. 92, 1131.

Phillips, M.L., Young, A.W., Scott, S.K., Calder, A.J., Andrew, C., Giampietro, V., Williams, S.C., Bullmore, E.T., Brammer, M., Gray, J.A., 1998. Neural responses to facial and vocal expressions of fear and disgust. Proc. R. Soc. London B Biol. Sci. 265, 1809-1817.
Phillips, M.L., Young, A.W., Senior, C., Brammer, M., Andrew, C., Calder, A.J., Bullmore, E.T., Perrett, D.I., Rowland, D., Williams, S.C., Gray, J.A., David, A.S., 1997. A specific neural substrate for perceiving facial expressions of disgust. Nature 389, 495-498.

Price, C.J., Friston, K.J., 1997. Cognitive conjunction: a new approach to brain activation experiments. Neuroimage 5, 261-270.

Rapcsak, S.Z., Comer, J.F., Rubens, A.B., 1993. Anomia for facial expressions: neuropsychological mechanisms and anatomical correlates. Brain Lang. 45, 233-252.

Rapcsak, S.Z., Galper, S.R., Comer, J.F., Reminger, S.L., Nielsen, L., Kaszniak, A.W., Verfaellie, M., Laguna, J.F., Labiner, D.M., Cohen, R.A., 2000. Fear recognition deficits after focal brain damage: a cautionary note. Neurology 54, 575-581.

Rolls, E.T., 1992. Neurophysiological mechanisms underlying face processing within and beyond the temporal cortical visual areas. Philos. Trans. R. Soc. London B Biol. Sci. 335, 11-20.

Rolls, E.T., 1999. The Brain and Emotion. Oxford University Press, Oxford

Rorden, C., Brett, M., 2000. Stereotaxic display of brain lesions. Behav. Neurol. 12, 191-200.

Schmolck, H., Squire, L.R., 2001. Impaired perception of facial emotions following bilateral damage to the anterior temporal lobe. Neuropsychology $15,30-38$.

Shah, N.J., Marshall, J.C., Zafiris, O., Schwab, A., Zilles, K., Markowitsch, H.J., Fink, G.R., 2001. The neural correlates of person familiarity. A functional magnetic resonance imaging study with clinical implications. Brain 124, 804-815.

Sprengelmeyer, R., Young, A.W., Schroeder, U., Grossenbacher, P.G., Federlein, J., Buttner, T., Przuntek, H., 1999. Knowing no fear. Proc. R. Soc. London B Biol. Sci. 266, 2451-2456.

Streit, M., Ioannides, A.A., Liu, L., Wolwer, W., Dammers, J., Gross, J., Gaebel, W., Muller-Gartner, H.W., 1999. Neurophysiological correlates of the recognition of facial expressions of emotion as revealed by magnetoencephalography. Brain Res. Cogn. Brain Res. 7, 481-491.

Talairach, J., Tournoux, P., 1988. Co-planar Stereotaxic Atlas of the Human Brain. Theime, Stuttgart, Germany.

Vuilleumier, P., Armony, J.L., Driver, J., Dolan, R.J., 2001. Effects of attention and emotion on face processing in the human brain. An event-related fMRI study. Neuron 30, 829-841.

Whalen, P.J., Rauch, S.L., Etcoff, N.L., McInerney, S.C., Lee, M.B., Jenike, M.A., 1998. Masked presentations of emotional facial expressions modulate amygdala activity without explicit knowledge. J. Neurosci. $18,411-418$

Winston, J.S., Strange, B.A., O'Doherty, J., Dolan, R.J., 2002. Automatic and intentional brain responses during evaluation of trustworthiness of faces. Nat. Neurosci. 5, 277-283.

Worsley, K., Marrett, S., Neelin, P., Vandal, A.C., Friston, K.J., Evans, A.C., 1996. A unified statistical approach for determining significant signals in images of cerebral activation. Human Brain Mapping 4, $58-73$.

Worsley, K.J., Friston, K.J., 1995. Analysis of fMRI time-series revisited-again. Neuroimage 2, 173-181.

Wright, C.I., Fischer, H., Whalen, P.J., McInerney, S.C., Shin, L.M., Rauch, S.L., 2001. Differential prefrontal cortex and amygdala habituation to repeatedly presented emotional stimuli. NeuroReport 12 , $379-83$.

Young, A.W., Aggleton, J.P., Hellawell, D.J., Johnson, M., Broks, P., Hanley, J.R., 1995. Face processing impairments after amygdalotomy. Brain 118, 15-24.

Young, A.W., Hellawell, D.J., Van De Wal, C., Johnson, M., 1996. Facial expression processing after amygdalotomy. Neuropsychologia 34, $31-$ 39.

Young, A.W., Rowland, D., Calder, A.J., Etcoff, N.L., Seth, A., Perrett, D.I., 1997. Facial expression megamix: tests of dimensional and category accounts of emotion recognition. Cognition 63, 271-313. 